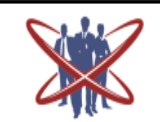

open access Jumal International Journal of Emerging Trends in Science and Technology

DOI: https://dx.doi.org/10.18535/ijetst/v7i9.01

\title{
Review on Heart Water Disease in Ruminants at Suro District
}

\author{
Authors \\ Dr Israel Gammada, Dr Wayu Jara \\ West Guji Zone, Suro Barguda Livestock and Fisher Office
}

\section{Summary}

Heart water is a tick borne infectious disease of ruminants affecting cattle, sheep, goats and antelope which is caused by the rickettsia organism cowdria ruminatium. The objective of this seminar is to review on heart water disease (cowdrosis) with the successful control of the disease. Heart water occurs only where vector ticks of the genus amblyomma are active and was limited in its occurrence to Africa, Madagascar and three countries in the West Indies. It is one of the main causes of death in imported breeds of cattle, sheep and goats in Africa south of the Sahara desert. A tentative diagnosis of heart water is based on the presence of amblomma vectors, of clinical nervous signs, and of transudates in the pericardium and thorax at postmortem examination, which must then be confirmed by demonstration of the causative organism or its DNA. Methods of control and prevention of cawdriosis is through chemotherapy, vaccination and vector control. Therefore calves of less than 4 weeks and lambs in the first week of life should be immunized.

Keywords: cawdriosis, Rickettsia, heart, ruminants, edema, amblomma vectors.
\end{abstract}

\section{Introduction}

Bovine heart water is an infectious and tick-borne disease of ruminants caused by the rickettsial organism, Ehrlichia ruminantium (formerly Cowdria ruminantium) and transmitted by ticks of the genus Amblyomma, particularly Amblyomma variegatum and Amblyomma habraeum which are widespread in Ethiopia (Mekonnen et al., 2001). Heart water is listed by the World Organization of Animal Health as a notifiable disease (OIE, 2014). Heart water is tick-borne diseases affects most of the world's cattle population and are widely distributed throughout the world, particularly in the tropics and subtropics. The complex of vector borne disease directly or indirectly constrains the livestock industry, which is of fundamental importance to rural people in sustaining not only their food supply but also their daily income and agricultural activity (Des Castro, 1997).

Video link:

http://www.youtube.com/watch? $v=U 5$ alNtwgz $2 E$

\&feature $=$ youtu.be

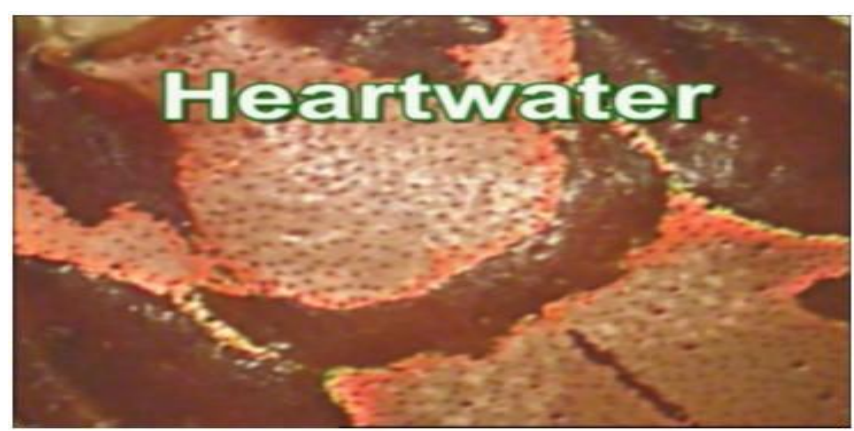

Figure 1: Diagrammatic representation of heart water. Source: (Allsopp et al, 2015)

Heart water is readily introduced into new regions in infected animals or ticks. Known and potential host ticks are widely distributed, and can be found on a variety of animals including reptiles. On at least one occasion, leopard tortoises and African spurred tortoises imported into Florida were found to be carrying infected ticks. Once the tick vector becomes established, eradication of heart water is difficult. One host tick, Amblyomma variegatum, was introduced into the Caribbean early in the $19^{\text {th }}$ century. 
During the 1970s and early 1980s, this tick spread rapidly from island to island; in some cases, it may have been carried by cattle egrets (OIE, 2015).

The most important clinical sign is high stepping gait and also severe diarrhea may be seen in some breeds of cattle. Especially in young's as field experience has shown, especially in the case of HW. The natural premunization of young animals under the protection of maternal colostral antibodies against the pathogen prevalent in the respective area is the best method to prevent the disease (Radotitis et al., 1994).

\section{Literature Review}

\subsection{Etiology}

Heart water results from infection by Ehrlichia (formerly Cowdria ruminantium) a small, Gram negative, pleomorphic coccus in the family Anaplasmataceae and order Rickettsiales. This organism is an obligate intracellular parasite. Strains of E. ruminantium are very diverse: while some strains are highly virulent, others appear to be non-pathogenic. Several different genotypes can co-exist in a geographic area, and may recombine to form new strains. These organisms can complicate the diagnosis of heart water, as cross reactions occur in all serological tests, and false positives have been reported in some PCR assays, depending on the primers used. This has implications for various aspects of heartwater, including its geographic distribution and the species affected (Allsopp BA, 2015).

Typically, the disease is characterized by high fever, nervous signs, hydro pericardium, hydrothorax and edema of the lungs and brain, and death. It is one of the major causes of stock losses in sub-Saharan Africa (Chamboko et al., 1999)

The disease is caused by Cowdria ruminantium, a rickettsia agent. it is the only species of the genus cowdria, in the tribe ehrlichia family Rickettisaceae, order Rickttsiales. The organism multiplies in vascular endothelial cells throughout the body and in the reticulum cells of the lymph nodes. The agent is small pleomorphic organisms $(0.2-2.7 \mathrm{~nm})$ and usually occurs in clumps from less than five to several thousand organisms within cytoplasm of infected capillary endothelial cells, especially in the brain. (bezuideshout et al.,1985)

\subsection{Epidemiology}

The epidemiology of heart water depends upon factors relating to the tick vector, the causative organism, and the vertebrate hosts. Important considerations relating to the tick vector are infection rates in the ticks, seasonal changes influencing tick abundance and activity, and the intensity of tick control. As far as the vertebrate hosts are concerned the availability of wild animal reservoirs and the age and genetic resistance of domestic ruminant populations are of importance (Mahan et al., 2001).

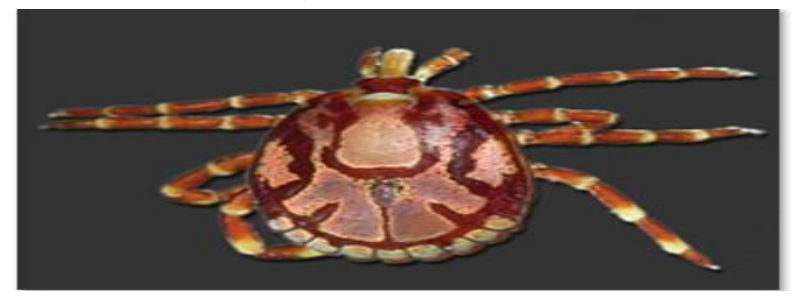

Figure 2: Photograph of Amblyomma hebraeum male that known by primary cause of heart water. Source: (Shkap, V et al, (2007)

\subsubsection{Geographic distribution}

Heart water occurs wherever ticks capable of transmitting the organism are present. The endemic area encompasses most of sub-Saharan Africa, including the islands of Madagascar, Sao Tomé, Reunion, Mauritius, and Zanzibar. The disease is absent from the Kalahari Desert and dry coastal areas of Namibia and South Africa. Heart water also occurs on the French Antillean islands of Guadeloupe and Antigua in the Caribbean Sea (Shkap et al., 2007)

Heart water occurs only where its Amblyomma tick vectors are present. Epidemiology depends on interaction of tick vector, causative agent, and vertebrate hosts. Tick vector: tick infection rates, seasonal changes influencing abundance and activity, and intensity of tick control. Causative Agent: differing genotypes affecting virulence or 
stimulation of cross protection. Vertebrate Host: availability of wild animal reservoirs, and age and genetic resistance. Because of its extreme fragility, the principal mode of bringing the disease into an area is by introduction of infected ticks or carrier animals (Coetzer et al., 2004)

\subsubsection{Species Affected}

E. ruminantium affects cattle, sheep, goats and water buffalo. It can also infect some wild ungulates, with or without clinical signs. Wild species proven to be susceptible to natural and/or experimental infection include blesbok (Damaliscus pygargus), wildebeest (Connochaetes gnou and C. taurinus), African buffalo (Syncerus caffer), eland (Taurotragus oryx), giraffes (Giraffa camelopardalis), greater kudu (Tragelaphus strepsiceros), sable antelope (Hippotragus niger), lechwe (Kobus leche kafuensis), steenbok (Raphicerus campestris), springbok (Antidorcas marsupialis), sitatunga (Tragelaphus spekii), Timor deer (Cervus timorensis), chital (Axis axis) and white-tailed deer (Odocoileus virginianus). Some other ungulates, including various cervids, bison (Bison spp.), and wild relatives of sheep and goats are also thought to be susceptible, but confirmation is lacking. Reports of possible fatal heartwater in an African elephant (Loxodonta africana) and a dromedary camel are unproven, and could have occurred from other causes (Bath GF et al., 2005)

\subsubsection{Transmission}

Heart water is transmitted by ticks of the genus Amblyomma. Most Amblyomma spp. are threehost ticks. Larvae and nymphs become infected when they feed on domestic and wild ruminants and possibly also on certain game birds and reptiles at a time when $E$. ruminantium is circulating in the blood of these hosts. The immature stages of the tick commonly feed on smaller species of domestic and wild ruminants and game birds, while the adults prefer cattle and the larger game animals, such as African buffalo (Allsopp et al ., 2005)

Heart water occurs only where its vectors are present and 10 Amblyomma spp. capable of transmitting the organism occurs in Africa. The major vectors are A. variegatum and A. hebraeum, the latter being the main vector of heart water in southern Africa. Amblyomma variegatum has the widest distribution in Africa and is the only originally African Amblyomma species that has established itself successfully outside the continent (on two islands in the French Antilles) (Chamboko et al., 1999)

\subsubsection{Prevalence}

It is a disease of Africa only with a variable prevalence no doubt related to the presence or absence of the tick vector. Although not now reported from North African countries, most of the rest of the continent is infected. In South Africa the incidence is quite high. there era no recent studies on the prevalence of heart water in different countries or regions of Africa but morbidity rate of $10 \%$ and case mortality of $50 \%$ have been reported in sheep and goats. A case mortality can be as high as $100 \%$ in per acute cases and as low as $0.10 \%$ in mild cases. The mortality rate is usually lower in cattle and the disease is less severe in indigenous breeds and related game animals reared in enzootic areas. Some of which may become symptom less carriers (Hall, 1998).

\subsubsection{Risk Factors and Immune Mechanisms}

Young animals have an age dependent innate resistance which is due to passive immunity derived from their dams. Recovered animals are immune to HW for 6 months to 4 years with possible exception of the Angora goat which is more difficult to immunize because of its very high susceptibility (Stewart, 2003).

\subsection{Pathogenesis}

The pathogenesis of heart water is not well understood. Vertebrate hosts are infected with E. ruminantium organisms through the saliva of attached ticks and/or by their regurgitated gut content. Initial replication of the organisms seems to take place in cells of the mononuclear phagocyte system in the regional lymph nodes, after which they are disseminated via the blood stream and invade endothelial cells of blood 
vessels in various organs and tissues where further multiplication occurs. In domestic ruminants E. ruminantium most readily infects endothelial cells of the brain, and this coincides with the onset of the febrile reaction (Shkap V et al., 2007)

Increased vascular permeability with transudation is responsible for effusion into body cavities and tissue edema, and this is particularly noticeable in the lungs, pericardial sac and pleural cavity. Edema of the brain is responsible for the nervous signs; hydro pericardium contributes to cardiac dysfunction during the terminal stages of the disease, and progressive pulmonary edema and hydrothorax result in eventual asphyxiation. It has been demonstrated that antibodies do not control the course of the disease. As E. ruminantium is an intracellular parasite it would be expected that CD8+ cytotoxic T-cells and CD4+ helper T-cells (Yonow et al., 1998)

\subsection{Clinical Signs}

Heart water occurs in four different forms per acute, acute and mild or sub clinical form determined by variations in susceptibility of the hosts and the virulence of various strains of the heart water agent. Per acute form of disease is usually seen in Africa in exotic breeds of cattle, sheep and goats induced to a heart water enzootic area. Heavily pregnant cows are especially prone to develop per acute disease.

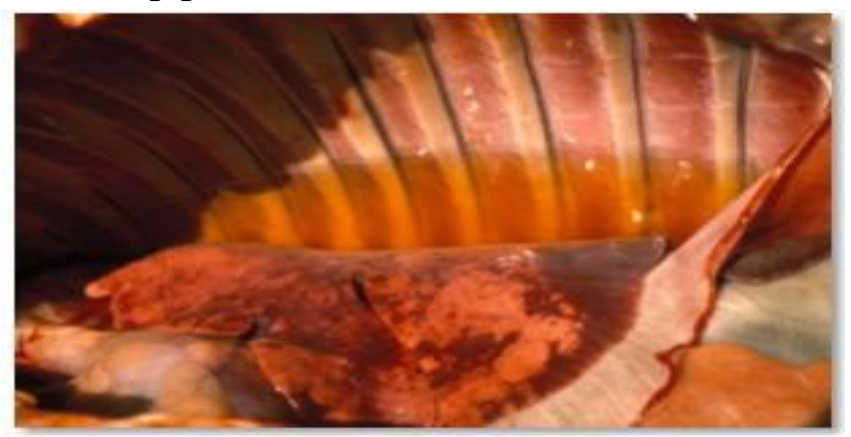

Figure 3: Severe hydrothorax in heart water showing accumulation of transparent transudate (bovine).Source: (Allsopp et al, 2015)

Sudden death occurs, usually preceded only by Fever, severe respiratory distress and terminal convulsions. Severe diarrhea may be seen in some breeds of cattle. The acute form of the disease the most commonly observed syndrome is seen in exotic and indigenous domestic ruminants a sudden fever of up to $107{ }^{0} \mathrm{~F}\left(42{ }^{0} \mathrm{C}\right)$ is followed by in appetence, depression, listlessness and rapid breath. Nervous signs then develop the tongue and circling, often with high stepping gait. Diarrhea is occasionally seen, especially in younger animals. The acute disease is usually total within a week of the onset of signs. Sub-acute form is characterized but prolonged fever, coughing a result of lung edema and mild in coordination, recovery or death occurs in 1 to 2 weeks. A mild or sub clinical form of the disease, known as "heart water fever" is seen in partially immune cattle or sheep in calves less than 5 weeks old in antelope and in some indigenous breed of sheep and cattle with high natural resistance to the disease. The case mortality rate in per acute cases is $100 \%$ in acute cases $50-90 \%$ in calves less than 4 weeks of age $5-10 \%$ whereas most animals recover in mild cases (Staeck et al., 2008 and Peinaar et al., 2006).

\subsection{Diagnosis}

Heart water occurs in four different clinical forms, determined by variations in host susceptibility, agent virulence and infective dose. Per acute disease is usually seen in Africa in non-native breeds of sheep, cattle and goats. Heavily pregnant cows are particularly susceptible to this form. Per acute disease is characterized by sudden death preceded by a brief interval of fever, severe respiratory distress, hyperesthesia, and lacrimation and, in some breeds of cattle, severe diarrhea. Terminal convulsions may be seen. This form of heart water is relatively rare (OIE, 2009).

Acute disease is the most common form of heart water in domesticated ruminants, and is seen in both nonnative and indigenous cattle, sheep and goats. Animals with the acute form of heart water usually die within a week. Disease begins with pyrexia, which may exceed $41^{\circ} \mathrm{C}$ within $1-2$ days after onset. It remains high for 4-5 weeks with small fluctuations and drops shortly before death, Fever is followed by in appetence, sometimes 
listlessness, diarrhea (particularly in cattle), and dyspnea indicative of lung edema (OIE, 2008).

Nervous signs develop gradually, and are generally less pronounced in sheep and goats than cattle. The animal is restless, walks in circles, makes sucking movements and stands rigidly with tremors of the superficial muscles. Cattle may push their heads against a wall or present aggressive or anxious behavior. In terminal stage the animal falls to the ground into lateral recumbence, pedaling and exhibiting opisthotonos, nystagmus, hyperesthesia, chewing movements, and frothing at the mouth. The animal usually dies during or following such an attack. Whole blood in anticoagulant:

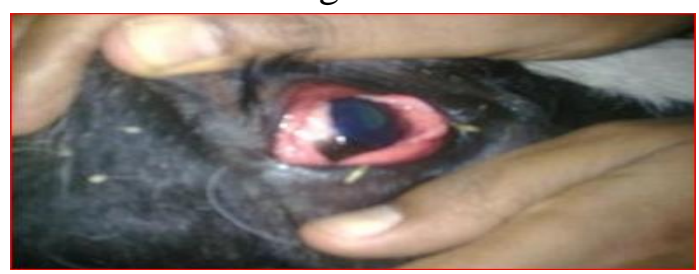

Figure 4: Animals showing congestion of the conjunctiva. Source: (Achenef et al., 2004)

In clinically ill animals, blood samples should be collected for PCR. PCR can sometimes detect organisms in the blood or bone marrow of carriers. For culture, blood is collected into an anticoagulant and diluted in culture medium: details are available in the OIE Terrestrial Manual. Samples should be kept refrigerated and shipped with ice packs. PCR can also be attempted on brain, lungs, kidneys, and thoracic fluids (Uilenberj and Camus, 1993).
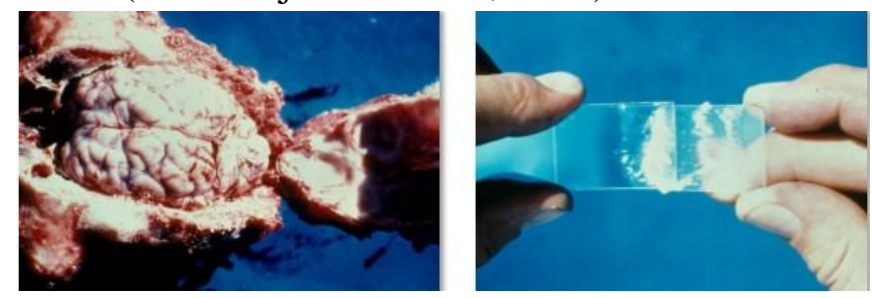

Figure 5: Removal of the brain for diagnosis of heart water is special technique to make a brain smear is necessary for the diagnosis of heart water. Source: (Mahan et al, 2001)

\subsection{Treatment of Heart water}

Treatment of heart water with antibiotics is only effective if animals are treated early at the onset of clinical symptoms and showed ineffectiveness when defined nervous symptoms occurred due to the acute nature of the disease, which does not always allow timely intervention to prevent a fatal outcome. In general, antibiotics of the tetracycline group (especially ox tetracycline) are the drugs of choice for successful treatment of heart water and will allow immunity to develop. Doxycycline and rifamycin are also very effective, and a wide variety of sulfonamides have successfully been used in heart water treatment. Treatment for ruminalatony, a commonly observed sequel to this disease, may be indicated, and diuretics may be useful to control fluid accumulations in body cavities (Mahan, et al, 2001

\subsection{Control of Heart Water}

As E. ruminantium cannot survive outside a living host for more than a few hours at room temperature, heart water is usually introduced into free areas by infected animals, including subclinical carriers, or by ticks. In heart water-free countries, susceptible ruminants from endemic regions are tested before importation. All animals that may carry Amblyomma, including nonruminant species, must be inspected for ticks before entry. Ticks may be carried into a country on illegally imported animals or migrating birds. Outbreaks are usually controlled with quarantines, euthanasia of infected animals and tick control (Brown et al, 2008).

Tick control has long been advocated as a means of controlling heart water. Even after the infected blood based vaccine was developed, tick control was still advocated as a supplementary or alternative means of control. Tick control can be either intensive or strategic, but intensive tick control has largely fallen into disuse. The main disadvantage is that animals may lose all immunity to tick-borne diseases because of the lack of a natural challenge. Strategic tick control implies the control of tick numbers so that natural infection of livestock occurs and high levels of immunity are maintained (Shkap et al, 2007).

During an outbreak, ticks should not be allowed to feed on infected animals. Iatrogenic transfer of 
blood between animals must also be avoided. In endemic regions, heart water can be prevented by tick control and vaccination. Animals moved into endemic areas may be protected by prophylactic treatment with tetracycline (WHO, 2009).

Several drugs have been used to treat animals suffering from heart water but the tetracycline, especially ox tetracycline, is the most widely used. Short-acting formulations of ox tetracycline are most commonly used at a dosage rate of 10 to 20 $\mathrm{mg} / \mathrm{kg}$ body weight, either administered intramuscularly as a single dose, or half the calculated dose is given intravenously and the other half intramuscularly. This treatment is usually repeated 24 hours later (Mahan et al., 2001).

\section{Conclusion and Recommendations}

Heart water (cowdrosis) is regarded as the most important tick-born disease in Africa countries. The most series losses are in exotic, susceptible cattle, sheep and goats that are imported in to areas in which the disease is enzootic. Though symptoms are often typical and even when nervous signs are present, many other diseases have to be considered for differential diagnosis. It will only occasionally be possible to make a precise diagnosis because of post mortem inspection of carcass. Based on the present review the following recommendations are forwarded:

\# Calves of less than 4 weeks and lambs in the first week of life should be immunized.

* Tick control has long been advocated as a means of controlling heart water.

Routine ox tetracycline injections may be used to protect susceptible animals against heart water when they are introduced into an endemic area.

To control ticks, use an approved insecticide on your cattle. Consult with your herd veterinarian for advice on approved and effective products.

It is also essential to inspect your livestock frequently for the presence of ticks and remove them promptly if found.
Acaricides are products aimed at killing ticks and should be applied to cattle every 2-4 weeks in affected areas.

Cattle in affected areas should be examine d daily, especially parts of the body difficult to gro om, for the presence of the Gulf Coast tick.

Pastures should be inspected weekly for the presence of the Gulf Coast tick.

\section{Acknowledgments}

First of all, I would like to praise almighty God for his unconditional help. Again I would like to thank my advisor Dr. Iyasu Ture for his constructive advices, guidance, material support and devotion of incalculable time in correction of this paper. In addition to this I would like to extend my thanks to all my friends and relatives who in one way or the other helped me during the seminar work. I would like to acknowledge to Jimma University College of agriculture and veterinary medicine for facilitating internet access and to all people who contribute information, advices and material.

\section{References}

1. Achenef M, Basaznew B, Mersha C, Wassie M and Gizat A, (2013).Veterinary Pharmacy and Biomedical Sciences Department.

2. Allsopp, B.A., Bezuidenhout, J.D., Prozesky, L. (2005). Heartwater, in: Infectious diseases of livestock, edited by Coetzer, J.A.W. \& Tustin, and R.C. Cape Town: Oxford University Press Southern Africa.

3. Bath G, van A, Pettey K. Control measures for some important and unusual goat diseases in southern Africa. Small Rumin Res. 2005; 60: 127-140.

4. Blood, D.C. and Radostitis (1994): Veterinary Medicine; A textbook of the diseae of cattle, sheep, pigs, goats and 
horses 8 ed., ELBS. BaillierTindasll.pp 1155-1156.

5. Brown and Skowronck, A. J. (1990).Histologic and immunochemical study of Pathogenesis of HW (Cowdriaruminantium infection) in goats and mice. American Journal of Veterinary Research, 51 (9): 1476-1480:17 Ref.

6. Brown.C and Torres .A. (2008). Foreign Animal Diseases, Seventh Edition.Commit tee of Foreign and Emerging Diseases of the US Animal Health Association.

7. Chamboko, T., Mukhebi, A.W., Callaghan, C.J.O., Peter, Kruska, R.L., Medley, G.F., Mahan, S.M., Perry, B.D. 1999. The control of heartwater on largescale commercial and smallholder farms in Zimbabwe. Preventive Veterinary Medicine, 39: 191-210.

8. Coetzer .J and Tustin.R.(2004). Infectious Diseases of Livestock, 2nd Edition.

9. De Castro J. Sustainable tick and tickborne disease control in livestock improvement in developing countries (1997).

10. Mahan S, Smit G, Kumbula D, Burridge, M, Barbet A, (2001). Reduction in mortalit $\mathrm{y}$ from heart water in cattle, sheep and goats exposed to field challenge using an inactivated vaccine. Veterinary Parasitology 97: 295-308.

11. Mahan, S.M., Smith, G.E., Kumbula, D., Burridge, M.J., Barbet, A.F. 2001. Reduction in mortality from heart water in cattle, sheep and goats exposed to field challenge using an inactivated vaccine. Veterinary Parasitology97: 295-308.

12. Mekonnen S, Hussein I, Bedane B (2001). The distribution of ixodid ticks in central Ethiopia. Under.J. Vet. Res. 68 (4):243251.

13. Shkap, V., De Vos, A.J., Zweygarth, E., Jongejan, F. 2007. Attenuated vaccines for tropical theileriosis, babesiosis and heartwater: the continuing necessity. Trends in Parasitology23: 420426.

14. World Organisation for Animal Health, (2008). - Terrestrial Animal Health Code. OIE, Paris.

15. World Organisation for Animal Health, (2009). - Terrestrial Animal Health Code. OIE, Paris.

16. World Organization for Animal Health (OIE, 2014). - Chapter 8.8. Heartwater.In Terrestrial Animal Health.

17. World Organization for Animal Health, (OIE, (2015).Manual of diagnostic tests and vaccines for terrestrial animals.

18. Yonow, T., Brewster, C.C., Allen, J.C., Meltzer, M.I. 1998. Models for heartwater epidemiology: practical implications and suggestions for future research. Onderstepoort Journal of Veterinary Research 65: 263-273

\section{Author Information}

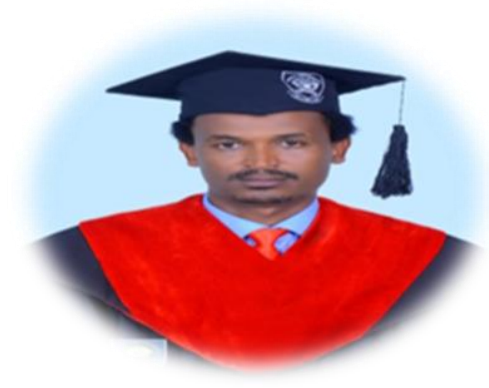

Name: Dr. Israel Gammada

Phone: +251926152824

Email:gamadaisrael@gmail.com

Address: Bulehora, Ethiopia

\section{Lists of Abbreviations}

CELISA...... Competitive enzyme, linked immunosorbent assay

HW............ heart water

IFA.............. indirect fluorescent antibody

PCR.............. polymerase chain reaction 